\title{
Sigmoid Colon
}

National Cancer Institute

\section{Source}

National Cancer Institute. Sigmoid Colon. NCI Thesaurus. Code C12384.

The portion of the colon that connects to the descending colon above and the rectum below. 